\title{
INCREASE SAFETY OF TRAFFIC CONTROL ON THE MINOR RAILWAY-TRACKS
}

The paper aims at detecting the existing safety risks on the minor railway-tracks being operated in accordance with the provision CD-D3 of the Czech Railways, and at providing the solutions and technologies suitable for the mentioned minor railway-tracks. These tracks are currently equipped with out of date safety related systems monitoring the railway, whose operation depends purely on human factor. The latest railway accidents were reported just on these low traffic frequency tracks.

\section{Introduction}

This study aims at providing the solutions and technologies for the minor railway-tracks currently operated according to the provision CD-D3 of the Czech Railways. The above mentioned tracks are characterized by a minimum number of safety related devices - as for the infrastructure, by the track offices not operated by the railway personnel and by the absence of the communication lines in the form of the track combined cables along the whole length of the railway track.

\section{Current State of Traffic Control on the Minor Railway-Tracks}

The current trend in the field of the safety related technologies is aimed at their development and the subsequent introduction in association with the main railway-tracks with the sufficient intensity of the railway traffic frequency. As for the economic aspect, the usage of these technologies on the low traffic frequency railway tracks is too expensive and non-profitable. As a result, these tracks are currently equipped with out of date safety related systems monitoring the railway, whose operation depends purely on human factor. The latest railway accidents were reported just on these low traffic frequency tracks.

Centralized controlling of the railway traffic on the integrated sequences or areas of minor tracks is not possible due to the currently used safety related systems. In order to be operated, this type of safety systems needs a high number of railway employees whose working capacity is not fully applied. Meanwhile, the maintenance of the outdated safety systems is time consuming and economically ineffective. Consequently, there comes the necessity of the low frequency railway track safety system innovation. With particular respect to the facts mentioned above, the philosophy and structure of the low frequency tracks safety system must be different from the system solutions used on the corridor tracks. The safety system solutions on such tracks can be basically approached in two different concepts.

- The first concept consists in building simple safety systems based on the principle of track permissions among the particular track offices, since frontal collisions of trains usually imply tragic consequences. To apply this concept, it is essential to provide and maintain the necessary infrastructure corresponding to the minor railway tracks i.e. laying cables (or using the cables already laid), and at least the minimal outside safety system (departure signal devices, axle counters etc.). With reference to the fact that the tracks, operated in accordance with the provision CDD3 of the Czech Railways, rank among those whose future is disputable (i.e. the tracks are being closed, their owners are being changed due to the assignments to private subjects, municipalities etc.), the present owner is not definitely guaranteed the infrastructure investment return. Since the afore-mentioned reasons and the analysis of the issue are not considered to be the subject matter of this study, they shall not be dealt with in the ongoing chapters.

- The second concept consists in transferring the safety system management from the infrastructure section to the vehicle section. Due to the considerable technical advance in the fields of telecommunications and new technologies based on satellite navigation, such concepts are prospective. The principle of these concepts is based on the fact that the directives and track permissions from the system operator are transmitted via available data networks directly to the vehicle in the front of the train, not to the safety devices of the track infrastructure. The currently used safety systems placed in the track offices are either operated or can even be removed. The solution proposed obviously provides the possibility of transferability to another track, in case the current track is temporarily closed. The aspects of such approach are dealt with in this study.

\footnotetext{
* Jan Slezak ${ }^{1}$, Juraj Spalek ${ }^{2}$

${ }^{1}$ Railway Research Institute, j.s.c., Czech Republic, E- mail: slezakj@cdvuz.cz

${ }^{2}$ Department of Control and Information Systems, University of Zilina, Slovakia
} 
The study is based on the basic distinction of the two terms "safety improvement" and "consequence elimination", which is a prerequisite of this study. While the notion of safety improvement assumes that the device itself eliminates the risk of danger leading to an accident, the notion of consequence elimination assumes that the consequences of the risk accrued are relieved by means of technologies i.e. stopping the vehicle, or more vehicles (trains) if any of the moving vehicles is involved in the accident with the track of another vehicle. In terms of safety, the study does not deal with the aspects eliminating the possible consequences, but it deals with the technologies enabling a real safety improvement. However the usage of factors leading to the consequence elimination is considered within the safety improving devices.

\section{Radio Block Descriptions}

Radio block is a technical device enabling controlling and monitoring of the railway traffic within the defined area in the form of authentic transport permissions reported to the leading railway vehicle (VDV), i.e. the driving and pilot vehicles by means of movable wireless network transferring data information and monitoring the railway vehicles track according to the permissions issued.

Radio block consists of the following subsystems:

RBC - radio block central

RBV - vehicle part of the radio block

Wireless transmission network

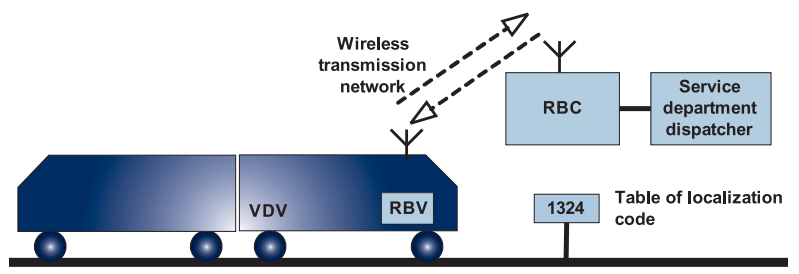

Fig. 1 Principle of the radio block system

According to the level of ability to monitor the vehicle movement by means of radio block, the radio-block devices can be categorized to several levels, while the lowest RB 0 provides the minimal functions as stated in the chapter 4 .

RB $0+$ level provides the functions equal to those of RB 0 The only difference detected consists in the fact that some of the safety critical functions (answer-back signal, vehicle answering and its movement in the area in compliance with the corresponding permission) performed by only human operation on the RB0 level, shall be completed by the functional check via the satellite navigation $(\mathrm{SN})$ on the $0+$ level.

\section{Basic Functions of the Basic Parts of the Radio Block}

\subsection{Radio Block Central}

- $\mathrm{RBC}$ acquires information on the state of the single elements and systems of the safety devices, it processes the information to the form applicable in the process of checking and controlling the travel of the vehicle, it secures the data authenticity conditions and their communication with the vehicle on the radio block via wireless network. The information on the state of the particular elements is provided by the safety system (YXL- station interlocking system, LX- level crossing).

- RBC performs the information authenticity check (in compliance with the EN 50 159-2) received from RBV via wireless transfer system, the information acquired is further evaluated and used in the process of controlling and securing the traffic.

- Displays the information acquired from RBV, YXL, LX, units on the operator's control board and enables to control the entire process of traffic.

\subsection{Vehicle Part of the Radio Block}

- $\mathrm{RBV}$ performs the authenticity control of the information received from the RBC via the specialized module

- RBV evaluates the information received from the RBC, safely displays its importance to the engine-driver and sufficiently ensures the vehicle travel monitoring with respect to the information received, all of the aforementioned is provided in the form of commands addressed to the vehicle control system.

- Receives the information on the vehicle position entered by the engine-driver via driver machine interface (DMI) within the vehicle localisation and transmits the information via the wireless data network to RBC.

- In the form of answer-back signals, the RBC provides the information necessary for cancelling the planned drives and for checking the availability of the particular track sections.

- RBC takes over the information on the movement (or waiting) of the vehicle acquired from the vehicle system.

- In the defined modes of the VDV, the RBC checks the availability of the key in the radio-block locks.

\subsection{Wireless Transmission Network}

- The network mediates the data communication between the RBC and RBV.

- It can as well provide voice connection between the operation point and the other mobile receivers, or among the mobile receivers themselves.

Note: In order to achieve voice connection, different wireless transmission networks can be used. If constructed, the communication connection on the unswitched network is used in case of wireless transmission network failure 
A coherent coverage by the wireless transmission network along the track in its whole length is not required by radio block. Data communication is required in track offices. The availability of the voice communication on the entire track as well, is not a condition of the traffic on the track. The date authenticity in compliance with the EN 50 159-2 shall not be ensured by the transmission network, but by the safety communication modules of the final receivers' devices.

\section{Possiblities of Radio Block Use}

The radio-block device of the RB $0+$ level is intended to use on the minor tracks characterized in the chapter 6 , it enables:

- Traffic safety improvement on the tracks equipped with the movement permission for VDV monitor - in the form of the vehicle movement permission delivery. The permissions are issued in $\mathrm{RBC}$ on the ground of the information provided by the safety devices (YXL, LX), the information from the driven vehicles and the commands given by the dispatcher on the utility point In case of the track offices not equipped with signalling devices, the permissions displayed to the engine driver on the DMI VDV replace the signals of the system signalling devices. If the system signalling devices are present on the track (signalling lights, flashing lights of the cross switches...) they are considered as effective.

- Displaying the reception and the range of the corresponding permission (to drive or to shift) in the form of a text message, and being confirmed by the engine driver, it enables the transition of the VDV to the appropriate mode of the vehicle movement.

- Completing the displayed permission by text messages on the restraints possibly occurring on the track the permission was issued for. The danger of neglecting the handover of the command is thus eliminated (as the information so far transmitted to the engine driver in written via RB 0 is transmitted in this way), and the RB qualities are considerably improved.

- Implementing at least a marginal test of the vehicle inquiring the vehicle conduct in accordance with the given commands. The vehicle command check consists in the following: VDV with an active RBV is allowed to move only upon an authorization of $\mathrm{RBC}$, i.e. after receiving and accepting the corresponding command on the vehicle. If there appeared a vehicle movement in the absence of such authorisation, the RBV issues the command stopping the vehicle. RBV does not check if the real vehicle speed corresponds to the actual speed limit, the speed limit in the particular track sequences permitted by the command is even not displayed to the engine-driver. The engine-driver follows the traffic signals or the track signalling devices, if present. In some case, when the different speed according to the track needs to be distinguished, the defined speed is transferred to the vehicle by means of restraints together with the permissions.

- The RB 0 level does not permit the check of the aim defined by the permission issued. Whereas, such a check is permitted by the RB0+ level within the extent as follows. In the SR (mod Staff Responsible) and IS (mod Isolation) module, and in case of vehicles without RBS, the currently transmitted data permission from RBC is commonly replaced by the dispatcher's voice permission - pursuant to the information on the VDV vehicles provided to RBC by the dispatcher.

- In respect of the $\mathrm{RB} 0+$, the $\mathrm{SN}$ monitors if the vehicle with an issued permission is moving in the area covered by the permission (MA-movement authority, SH-shunting, JOV- ride of the service rail-switch), and if the data on the vehicle position entered by the engine driver (answer-back signal) comply with the vehicle's position ensured by the $\mathrm{SN}$ devices. In case of any discrepancy, the engine driver is given a warning by an acoustic or optic signal.

- By means of the RBC point the vehicle movement is monitored via the vehicle localisation referred by the engine driver entering the numerical code of the position (number of the localisation section). With respect to the answer back signals, the automatic cancel of the given drives on the RBC.

- Issuing an emergency command to stop the VDV by the RBC, this serves as a prompt solution of stopping the vehicle in case of an impending danger.

- Minimizing the needs of the safety system infrastructure parts by means of providing the technical check of the lockable crossswitches key in the particular track offices in the RBC via the lock on the VDV checked in the RBV.

\section{Characteristics of the Tracks Suitable for the Radio Block Usage}

The radio-block technical devices must be selected in the way enabling the radio-block application on the tracks conforming to the characteristics as follows:

- Single track line in an arbitrary traction.

Recommendation: The drive in the controlled sequence is enabled mainly by the VDV equipped with RBV. The vehicles not equipped with the RBV system are allowed only exceptionally.

- The traffic before the RB implementation is simple, defined by minimal over-taking, the shifts being secured by the train crew.

- The maximum number of the track offices in the area controlled by one RBC unit is 35 , whereas there is a possibility of integrating up to 35 offices from four different tracks, however there shall be the safe traffic organisation among the given track within one RBC unit secured.

- Track speed: RB0 - max. $60 \mathrm{~km} / \mathrm{h}, \mathrm{RB} 0+-\max .90 \mathrm{~km} / \mathrm{h}$. The given speed limit may be restricted due to the construction and the cross-switches criteria and safety parameters.

- The number of VDV signed up in the system (within one RBC unit) is max. 16

- The monitored area (sequences between the stations, station gridiron and the track point disposal's tracks) does not imply the necessity of being equipped with the signalling devices. In case any of these track sequences are equipped with the signalling devices, these can be integrated in the RBC functions.

- The trains shall not necessarily be equipped with the devices securing the vehicle integrity, however such devices are recommended. 
- In case the train involves more driving engines, or one or more control engines, only one of the driving engines shall be considered VDV and therefore it shall be controlled by the RBC.

- VDV unit shall be equipped with the KBS (vigilance of driver) device providing the edge to the electromagnetic valve of the service brake.

Note: RB 0 ad RB0+ is therefore intended for the tracks used with respect to the provision $\mathrm{CD}-\mathrm{D} 3$, while the usage of $\mathrm{RB} 0+$ is recommended.

\section{Radio Block Principles}

The voice communication between the engine driver and the operating dispatcher is replaced by the secured wireless data communication of the terminal points - RBC and RBV, which brings the elimination of errors in the process of the data transmission and reception.

The RBC monitors the dispatcher's activity and disables the usage of conflicting driving tracks. GDV modifications, delays, changes in the train crossings do not have any impact on safety, while the RBC can command and check the external interlocking systems factors.

RBV further monitors the engine driver's activity and disables the drive (shift) of the VDV without RBC permission. It provides a continuous display of the permission including the LX restraints in the field of vision. RBV addresses warnings to the engine driver in case the engine leaves the authorised section defined in the given permission (the train is stopped) and finally the RBV checks the state of the cross-switch keys.

Principle of assignment movement authority and conferment of an answer - back signal from train is illustrated on the figure number 2. Chronology of award procedures is obvious from the numbers in the picture.

\section{Conclusion}

The newly proposed RB system complying with the provision CD-D3 of the Czech Railways represents a higher level of traffic safety standards. The RBC and RBV devices are examples of safe traffic. The dispatcher's activity is continuously monitored by the system (in the course of the data entering, through driving the

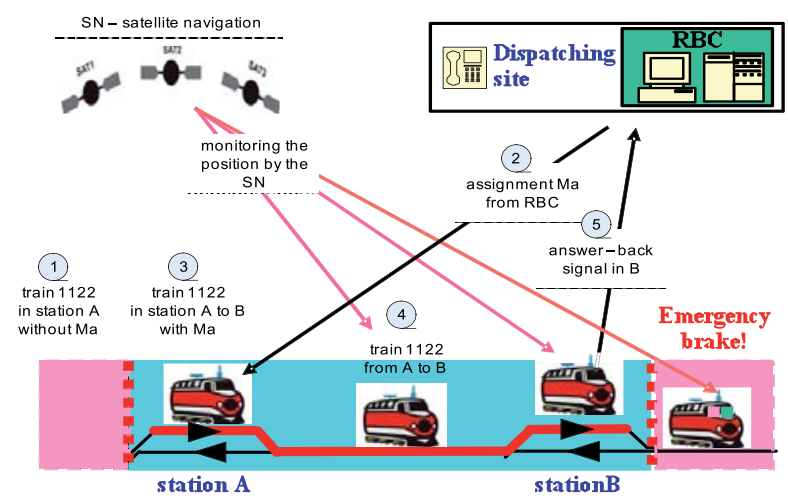

Fig. 2 Principle of assignment movement authority and conferment of an answer - back signal from train

engine, shifting...). The communication between the dispatcher and the engine driver as provided by the RB system shall be realised mainly as data communication (the main technical medium of the traffic safety systems as defined by the provision CD-D3 consists in voice communication between the dispatcher and the engine driver by means of wireless network directly from the engine cabin, or on the line from the track office). The proposed RB system, as well as communication in the system, considerably contributes to the general traffic safety improvement.

\section{List of Abbreviations Used in This Publication}

CD-D3 provision of the Czech Railways

DMI driver machine interface

IS mod of system radio block - Isolation

JOV mod of system radio block - ride of the service rail-switch

KBS vigilance of driver

LX level crossing

MA movement authority

RB system radio block

$\mathrm{RBC}$ radio block central

RBV vehicle part of the radio block

SH mod of system radio block -shunting

SN satellite navigation

SR mod of system radio block - Staff Responsible

VDV leading railway vehicle

YXL station interlocking system

\section{References}

[1] Czech Railways: Provision of Simplified Control of the Railway Traffic, CD-D3, Official edition, Olomouc: JERID, 1997.

[2] FRYBORT, Fr.: Definition of System and Conditions of Use RB 0+, version 1.05, AZD Prague, 2007.

[3] SLEZAK, J.: Safety Conception of RB-net, version 1.13, VUZ Prague, 2009.

[4] ROFAR, J., FRANEKOVA, M., HOLECKO, P.: Modelling of Safety Characteristics of Redundant Safety-Related Transmission System via Markov's Analysis, Communications - Scientific Letters of the University of Zilina, Vol. 12, No. 3a, pp. 93-96, ISSN 1335-4205.

[5] RASTOCNY, K., FRANEKOVA, M.: Modelling in Development of Safety-related Communication Systems, Communications - Scientific Letters of the University of Zilina, No. 1, 2008, pp. 24-30, ISSN 1335-4205. 